This is an electronic reprint of the original article. This reprint may differ from the original in pagination and typographic detail.

Author(s): Miettinen, Kaisa; Podkopaev, Dmitry; Ruiz, Francisco; Luque, Mariano

Title: A new preference handling technique for interactive multiobjective optimization without trading-off

Year: $\quad 2015$

Version:

Please cite the original version:

Miettinen, K., Podkopaev, D., Ruiz, F., \& Luque, M. (2015). A new preference

handling technique for interactive multiobjective optimization without trading-off.

Journal of Global Optimization, 63(4), 633-652. https://doi.org/10.1007/s10898-015-

0301-8

All material supplied via JYX is protected by copyright and other intellectual property rights, and duplication or sale of all or part of any of the repository collections is not permitted, except that material may be duplicated by you for your research use or educational purposes in electronic or print form. You must obtain permission for any other use. Electronic or print copies may not be offered, whether for sale or otherwise to anyone who is not an authorised user. 


\title{
A New Preference Handling Technique for Interactive Multiobjective Optimization without Trading-Off
}

\author{
Kaisa Miettinen ${ }^{1}$, Dmitry Podkopaev ${ }^{2,3}$, Francisco Ruiz ${ }^{4}$, and Mariano Luque ${ }^{4}$ \\ ${ }^{1}$ University of Jyvaskyla, Department of Mathematical Information Technology, P.O. \\ Box 35 (Agora), FI-40014 University of Jyvaskyla, Finland, Tel.: +358 50373 2247, \\ Fax: +358 14260 2771, kaisa.miettinen@jyu.fi \\ ${ }^{2}$ University of Jyvaskyla, Department of Biology and Environmental Science, P.O. Box \\ 35 (Ambiotica), FI-40014 University of Jyvaskyla, Finland \\ ${ }^{3}$ Department of Intelligent Systems, System Research Institute of the Polish Academy of \\ Science, Newelska 6, 01-447 Warsaw, Poland (current address) \\ ${ }^{4}$ Department of Applied Economics (Mathematics), Universidad de Málaga, Calle Ejido \\ 6, 29071 Málaga, Spain
}

March 13, 2015

\begin{abstract}
Because the purpose of multiobjective optimization methods is to optimize conflicting objectives simultaneously, they mainly focus on Pareto optimal solutions, where improvement with respect to some objective is only possible by allowing some other objective(s) to impair. Bringing this idea into practice requires the decision maker to think in terms of trading-off, which may limit the ability of effective problem solving. We outline some drawbacks of this and exploit another idea emphasizing the possibility of simultaneous improvement of all objectives. Based on this idea, we propose a technique for handling decision maker's preferences, which eliminates the necessity to think in terms of trade-offs. We incorporate this technique into an interactive trade-off-free method for multiobjective optimization. We call the resulting method NAUTILUS 2, which is also suitable for negotiation support. We demonstrate the applicability of the new method with an example problem.
\end{abstract}

Keywords: multiple objectives, interactive methods, preference information, NAUTILUS method, negotiation support

\section{Introduction}

By solving a multiobjective optimization problem we typically understand finding a feasible solution which satisfies the decision maker (DM) in the best way (see e.g. Miettinen (1999)). Such a solution is often referred to as the most preferred solution and is determined based on the DM's preference information. A widely used class of solution methods in multiobjective optimization is interactive methods (see, e.g., Branke et al. (2008); Luque et al. (2011); Miettinen (1999); Miettinen et al. (2008); Ruiz et al. (2012); and references therein). They iteratively proceed towards the most preferred solution allowing the DM to learn about the interdependencies among the objectives during the solution process and adjust one's preferences accordingly. Interactive methods usually generate feasible solutions (to be called solution candidates) based on preference information provided by the DM, and when the DM changes preferences, a new or some new solution candidates are derived.

Most interactive methods concentrate on Pareto optimal solution candidates, where none of the objective function values can be improved without impairing at least one of the others. This concentration is natural as one can assume that the most preferred solution 
is Pareto optimal. Limiting the consideration in this way has some advantages, such as significant reduction of the set of solution candidates, and also the possibility to benefit from the theory of vector optimization. However, the analysis of solution processes from the human perspective reveals some disadvantages, mostly because the concept of Pareto optimality is strongly related to the concept of trade-off between objectives (referred often to as conflicting objectives).

In this paper, our starting point is the same as in Miettinen et al. (2010) where the interactive NAUTILUS method was proposed. We develop an approach to multiobjective optimization, which is free from the necessity of considering trade-offs. We get rid of thinking in terms of trade-offs both when handling DM's preferences and guiding the DM through the set of solution candidates. In order to introduce this in practice, we propose a new preference handling technique which is easily understandable for the DM and allows the DM to conveniently control the solution process. With examples we demonstrate the easy controllability compared to the approaches proposed in connection with the NAUTILUS method. As a concrete example of how the new preference handling technique can be used, we incorporate it in the interactive NAUTILUS method and the resulting method is called NAUTILUS 2. It is a practical trade-off-free approach to solving multiobjective optimization problems. At each iteration, we find a solution that simultaneously improves all the objectives when compared to the previous iteration. Therefore, the DM will always be more satisfied with any new iteration. Thanks to the new preference handling technique, the DM can conveniently control the improvement ratios among the objective functions and only the final solution is Pareto optimal. This progressively satisficing technique can be seen to connect our approach with Simon's models of rational choice (Simon, 1956).

The new preference handling technique can be also useful in negotiations when a group of decision makers must reach a common decision. In this case, at each iteration, DMs negotiate on the direction of simultaneous improvement of objectives. This is a fruitful setting for negotiations when each DM can gain instead of having to sacrifice.

There are few interactive approaches that start from an inferior point and make progress towards the Pareto optimal set by simultaneously improving objective values. However, in these works no attention has been paid to ratios of improvement when expressing preferences by the DM. Among such methods, besides the already mentioned NAUTILUS method (Miettinen et al., 2010), it is worth mentioning the algorithm by Arbel and Korhonen (1996) (designed for linear problems only) and an interactive method of negotiation support by Ehtamo et al. (2001).

This paper is organized as follows. In the next section, we formulate a general multiobjective optimization problem and briefly consider weaknesses of interactive methods based on considering Pareto optimal solution candidates during the solution process. Based on this motivation, in Section 3 we develop the preference handling technique which is free from the thinking in terms of trade-offs and demonstrate its strengths when compared to techniques proposed earlier. This supports the DM's easy controllability of expressing preferences with the new preference handling technique. In Section 4, we present two examples of how the preference handling technique can be used. Firstly, we incorporate the technique into a trade-off-free interactive method to be called NAUTILUS 2. Secondly, we present a numerical example to illustrate the applicability of the preference handling technique and the method. Finally, we draw some conclusions in Section 5. 


\section{Concepts and Consideration of Pareto Optimality}

We consider multiobjective optimization problems formulated as follows:

$$
\begin{array}{ll}
\text { minimize } & \mathbf{f}(\mathbf{x})=\left(f_{1}(\mathbf{x}), \ldots, f_{k}(\mathbf{x})\right)^{T} \\
\text { subject to } & \mathbf{x} \in S
\end{array}
$$

where the DM wishes to simultaneously minimize $k(k \geq 2)$ objective functions $f_{i}: S \rightarrow \mathbb{R}$. The decision vectors $\mathbf{x}=\left(x_{1}, \ldots, x_{n}\right)^{T}$ belong to the feasible set $S$, which is a nonempty compact subset of $\mathbb{R}^{n}$. The image of the feasible set is called the feasible objective set $\mathbf{f}(S)$ and its components $\mathbf{z}=\mathbf{f}(\mathbf{x})=\left(f_{1}(\mathbf{x}), \ldots, f_{k}(\mathbf{x})\right)^{T}$ consisting of objective (function) values are called objective vectors. By a DM we refer to a person who knows the problem to be solved and can specify preference information related to it.

For any two vectors $\mathbf{v}, \mathbf{w} \in \mathbb{R}^{k}$, we say that $\mathbf{v}$ dominates $\mathbf{w}$ if $v_{i} \leq w_{i}$ for all $i=1, \ldots, k$ and $\mathbf{v} \neq \mathbf{w}$. The set of Pareto optimal decision vectors is defined by

$$
E=\left\{\mathbf{x} \in S: \text { there is no } \mathbf{x}^{\prime} \in S \text { such that } \mathbf{f}\left(\mathbf{x}^{\prime}\right) \text { dominates } \mathbf{f}(\mathbf{x})\right\} .
$$

We use the term Pareto optimal solution to refer either to an element of $E$ or $\mathbf{f}(E)$ (the image of $E$ in the objective space), which will be clear from the context. The set $\mathbf{f}(E)$ is called the Pareto optimal set.

It is often desirable to have some information about the ranges of the objective function values in the Pareto optimal set. The ideal objective vector and the nadir objective vector are defined, respectively, as $\mathbf{z}^{\star}=\left(z_{1}^{\star}, \ldots, z_{k}^{\star}\right)^{T}$ such that $z_{i}^{\star}=\min _{\mathbf{x} \in E} f_{i}(\mathbf{x})$ for $i=1, \ldots, k$, and $\mathbf{z}^{\text {nad }}=\left(z_{1}^{\text {nad }}, \ldots, z_{k}^{\text {nad }}\right)^{T}$ such that $z_{i}^{\text {nad }}=\max _{\mathbf{x} \in E} f_{i}(\mathbf{x})$ for $i=1, \ldots, k$. That is, the components of the ideal and the nadir objective vectors are, respectively, the best and the worst values that each objective function can achieve in the Pareto optimal set. While the ideal objective vector can be obtained by separately minimizing each of the objective functions in the feasible set, the nadir objective vector is, in general, more difficult to obtain and typically one needs to settle for approximations by using, e.g., payoff tables (see Miettinen (1999) and references therein). Lately, some approaches for more reliable nadir objective vector generation have been proposed (see, e.g., Deb et al. (2010); Szczepanski and Wierzbicki (2003)).

Solving problem (1) means finding the most preferred decision vector (also referred to as the most preferred solution), i.e., an element of $S$ which is the most satisfying for the DM. It is rational to assume that if one solution dominates another solution, the DM always prefers the former to the latter. Therefore in multiobjective optimization, the search for the most preferred solution is constrained to Pareto optimal solution set (Miettinen, 1999; Branke et al., 2008). We concentrate on interactive methods because they are regarded as the most promising methods of multiobjective optimization due to numerous advantages (Miettinen, 2002; Miettinen et al., 2008; Miettinen and Hakanen, 2009). Broadly speaking, an interactive method consists of repetitive iterations of generating Pareto optimal solution candidates based on preference information provided by the DM. In general, at each iteration the DM considers solution candidates and, by expressing preferences, indicates how the candidates should be changed to get more preferred solution candidates. Solution processes facilitated by such methods involve moving from one Pareto optimal solution to another and comparing solution candidates by the DM.

As mentioned in the introduction, the starting point of this paper in questioning the idea of dealing with Pareto optimal solutions only throughout the solution process of an interactive method is the same as in Miettinen et al. (2010). Here, we briefly summarize the reasons. According to the definition of Pareto optimality, moving from one such 
solution to another one in order to improve the value(s) of some objective function(s) necessitates impairment of the value of some other objective function(s). The consequences of such changes cannot be always easily assessed by the DM because of human biases, as shown in the prospect theory developed by Kahneman and Tversky (1979). They discovered that humans' attitudes to losses loom larger than gains. For example, the pleasure of gaining a sum of money is shown to be lower than the dissatisfaction of losing the same amount of money. Thus, requiring the DM to trade-off may hinder her/his willingness to move from the current Pareto optimal solution.

Further studies have demonstrated negative effects of trading-off such as decisional stress (Janis and Mann, 1977), decisional conflict and as a result, less accurate decision making (Aloysius et al., 2006), as well as too small numbers of iterations in interactive methods (Korhonen and Wallenius, 1996). The latter phenomenon observed by Gardiner and Vanderpooten (1997) can be attributed to anchoring (Miettinen, 1999; Buchanan and Corner, 1997), where the DM fixes one's thinking on some (possible irrelevant) information which hinders his/her willingness to move from the current Pareto optimal solution.

The considerations concerning negative effects of trading-off and anchoring can be applied also in the case of group decision making, where several DMs with possibly conflicting interests try to find a satisfactory solution for all of them. Trading-off between interests of different persons may cause more substantial conflicts than just psychological issues of a single DM. The anchoring effect influencing any of the group members affects the whole group. Behavioral issues of a group of DMs are more complicated and have not received so much attention as the case of a single DM. Some analysis can be found in Tsay and Bazerman (2009).

Summing up, forcing the DM to trade-off among objectives and iterate among Pareto optimal solutions only is not always advisable. If, on the other hand, the DM starts from a point where improvement in all objectives is possible, the DM can direct the solution process more freely always attaining only gains. Indeed, if the DM first sees a very unsatisfactory solution, a somewhat better solution is regarded more satisfactory than otherwise.

\section{$3 \quad$ Preference Handling Technique}

Even though the concept of conflicting objectives implying the need of trade-offs is widespread in the multiobjective optimization literature, the DM may perceive multiple objectives in decision making problems not as conflicting, but as mutually supportive if one starts from an unsatisfactory point. Indeed, it follows directly from the multiobjective optimization problem statement that all the objectives are to be minimized simultaneously, rather than some of them having to be improved at the expense of deteriorating other ones. Moreover, in many practical problems, there are certain proportions in which the objectives should be improved to achieve the most intensive synergy effect. Here, we propose the idea of the most promising direction of simultaneous improvement of objectives which agrees with the well-known assumption of concavity of the utility function (Guerraggio and Molho, 2004), implying that this function grows faster in certain directions of simultaneous decrease of objective function values (starting e.g. from the nadir objective vector).

The idea of a trade-off-free approach calls for a new way of expressing preferences. Once the DM knows that all objectives can be simultaneously improved, the question is how does (s)he want them to be improved. We propose to represent the DM's preferences as a direction of simultaneous improvement of objectives. Expressing such kind of aspiration 
does not necessitate knowledge about the Pareto optimal set and, thereby, can be used in the learning phase of interactive methods (cf. Miettinen et al. (2008)) even before any Pareto optimal solutions are available. However, DM's preferences expressed in this way can be combined with the Pareto optimality concept, as the DM is aiming at a Pareto optimal solution as the final one. An approach to dealing with such preferences was presented in Podkopaev and Miettinen (2011). We use this to develop a preference handling technique for implementing a trade-off-free method to support decision making.

\subsection{Expressing preferences}

Some ways for expressing preferences in trade-off-free environments have already been proposed. In Luque et al. (2009) and Miettinen et al. (2010), two ways have been proposed which are based on the importance given by the DM to the improvement of each objective:

- Rank the objectives according to the relative importance of improving each current objective value. This importance evaluation allows to allocate the $k$ objective functions into index sets $J_{r}$ which represent the importance levels $r=1, \ldots, s$. If $r<t$, then improving the current objective function values in the index set $J_{r}$ is less important than improving the current objective function values in $J_{t}$.

- Specify percentages reflecting how the DM would like to improve the current objective values, by answering to the following question: Assuming you have one hundred points available, how would you distribute them among the current objective values so that the more points you allocate, the more improvement on the corresponding current objective value is desired?

These ways of expressing preferences have two major drawbacks. Firstly, given that the DM is asked about the relative importance of improving each objective, the quantification of this information may not be straightforward. Besides, we also need to normalize the numbers obtained according to the ranges of the objective function values in the Pareto optimal set. For example, in the former case, all objectives belonging to the index set $J_{r}$ are assigned the weight

$$
\mu_{i}=\frac{1}{r\left(z_{i}^{\text {nad }}-z_{i}^{*}\right)} \quad\left(i \in J_{r}\right)
$$

In the latter case, if $p_{i}$ points are given to the objective function $f_{i}$, then $\Delta q_{i}=p_{i} / 100$ and

$$
\mu_{i}=\frac{1}{\Delta q_{i}\left(z_{i}^{\text {nad }}-z_{i}^{*}\right)} \quad\left(i \in J_{r}\right)
$$

Although the information required in these two ways may be regarded as easy to be provided by the DM, there is a certain degree of arbitrariness in the election of the parameters that are used to build the weights, and this can result in undesired effects when determining the corresponding solutions. Secondly, many authors argue that weighting objective functions based only on the psychological notion of importance is one of the most common critical mistakes when modelling preferences (see, e.g. Keeney (1996)).

In order to avoid the drawbacks and possible inconsistencies described above, in this paper, we propose a preference model specification based on actual values of the objective functions and comparisons among them. The way we propose for defining a direction of simultaneous improvement of objectives consists of a starting point in the objective space and a vector representing a direction of improvement. In terms of problem (1), the starting point is defined by $\mathbf{s} \in \mathbb{R}^{k}$ and the direction by $\delta \in \mathbb{R}^{k}$. Although it is not required for the starting point to be a feasible objective vector, it is assumed that $\mathbf{s}$ is 
meaningful for the DM. In other words, s represents some hypothetical objective vector, which can be evaluated by the DM on the basis of his/her expertise and preferences.

The information represented by $\mathbf{s}$ and $\delta$ is interpreted as follows: the DM wants to improve the starting point $\mathbf{s}$ as much as possible, decreasing the objective function values in proportions of $\delta$. By setting $\delta_{i}>0$ for $i=1, \ldots, k$ we emphasize the fact that the DM wants to improve all the objective values simultaneously.

The DM can select the starting point keeping in mind that the final objective vector should have smaller values in all components, thus, there must be space for improvement in all components. Another possibility is to set the nadir objective vector as the starting point ensuring that any Pareto optimal objective vector is initially a possible improvement.

There are different approaches to express preferences in order to define the direction of improvement. The following three approaches were outlined in Podkopaev and Miettinen (2011).

(i) The DM sets the values $\delta_{1}, \ldots, \delta_{k}$ as components of $\delta$ directly. This is possible when the DM understands the idea of the preference model and can operate with objective function values in his/her mind.

(ii) We define the improvement ratio between two different objectives $f_{i}$ and $f_{j}$ as $\theta_{i j}=$ $\delta_{j} / \delta_{i}$. This ratio has the following meaning: the DM wishes that the improvement of $f_{i}$ by one unit should be accompanied with the improvement of $f_{j}$ by $\theta_{i j}$ units. Here, the DM selects an objective $f_{i}(i=1, \ldots, k)$ and for each of the other objectives $f_{j}$ sets the value $\theta_{i j}$. Then the direction of improvement is defined by $\delta_{i}=1$ and $\delta_{j}=\theta_{i j}, j \neq i$.

(iii) As a generalization of the approach (ii), the DM sets values of improvement ratios freely for some selected pairs of objective functions. In what follows, we describe a procedure supporting the DM in doing that, which includes a mechanism ensuring that improvement ratios set by the DM define the components of the vector $\delta$ completely and consistently.

Note that these approaches of providing directions of improvement are based on objective function values, rather than considerations about the importance of each objective. Therefore, these new approaches do not share the drawbacks described earlier in this section that are valid for the ways proposed in Luque et al. (2009) and Miettinen et al. (2010). The first two approaches are easy to implement. The third, the most flexible approach, requires an interactive procedure that guides the DM in expressing preference information while ensuring that this information fully and consistently defines a direction of improvement. Below we propose such a procedure.

To give further details of the approach (iii), let us assume that improvement ratios have been set by the DM for some pairs of objectives. Let us denote this information by $\Theta$ and the ratio set for a pair of objectives $(i, j)$ by $\theta_{i j}$. We construct an undirected graph $G$ corresponding to $\Theta$ as follows: the set of nodes is the set of indices of objectives $V=\{1, \ldots, k\}$; the set of edges contains those and only those index pairs $\{i, j\}$, for which the DM has set the value of $\theta_{i j}$. Note that setting the value of $\theta_{i j}$ implies also setting the value of $\theta_{j i}=1 / \theta_{i j}$ and, therefore, our graph is undirected. Obviously, in order to be able to obtain a vector $\delta$ from information $\Theta$, it is necessary and sufficient that $G$ contains a spanning tree of the complete graph on $k$ vertices of $V$ (denoted by $K_{k}$ ). Then, one can obtain $\delta$ by the following procedure.

Procedure 1: Set $\delta_{i}=1$ for some arbitrary $i=1, \ldots, k$. Use a tree search algorithm (e.g. depth- or breadth-first, cf. Cormen (2009)) starting from $i$ in order to visit each 
node $j$ of the spanning tree and set $\delta_{j}=\delta_{p} \theta_{p j}$, where $p \in V$ is a previously visited node connected with $j$.

Observe that although the components of $\delta$ depend on the starting point of the tree search, it is easy to see that the ratios between any of them do not. This means that once $G$ is a spanning tree of $K_{k}$, the existing information $\Theta$ uniquely determines improvement ratios between the rest of the pairs of different objectives.

Keeping the above considerations in mind, let us outline an interactive procedure implementing the approach (iii) for defining the direction of improvement $\delta$. Setting improvement ratios by the DM is accompanied by adding corresponding edges to $G$. The aim of the procedure is guiding the DM in setting improvement ratios such that $G$ will become a spanning tree of $K_{k}$. Therefore, in the procedure it is ensured that $G$ remains a forest (disjoint union of trees). For this purpose, a set $P$ is introduced which contains all pairs of objectives such that adding to $G$ an edge corresponding to any of these pairs does not create a cycle. The DM is allowed to set improvement ratios only for pairs in $P$.

The procedure is initialized by setting $G=(V, \emptyset)$, where $V=\{1, \ldots, k\}$ and $P=$ $V \times V \backslash\{(1,1),(2,2), \ldots,(k, k)\}$. At each iteration of the procedure:

- Present the set $P$ to the DM.

- Ask the DM to select a pair of objectives $(i, j)$ from $P$ and to provide a value $T>0$ as the desirable improvement ratio of this pair.

- Add the edge $\{i, j\}$ to $G$, set $\theta_{i j}$ to $T$ and $\theta_{j i}$ to $1 / T$.

- If the number of edges in $G$ equals $k-1$, then $G$ is a spanning tree of $K_{k}$, which means that the procedure is completed. Otherwise, remove all pairs of vertices where one vertex is either $i$ or $j$ and the other one is some other vertex of the same tree ${ }^{1}$ from $P$ and repeat the iteration of the procedure.

Once the graph is a spanning tree of $K_{k}$, the direction of improvement is obtained as described in Procedure 1.

The proposed procedure of setting improvement ratios looks more complicated than it really is for the DM, if it is appropriately implemented with a user-friendly interface. The electronic attachment "direction setting interface.cdf" demonstrates the implementation of this procedure for the example described below in Computable Document Format $(\mathrm{CDF})$. Its interface includes a table of input fields for entering improvement ratios for all allowed pairs of objectives. The directed version of graph $G$ is drawn and automatically updated, where the directions correspond to pairs of objectives selected by the DM. Besides ratios directly set by the DM, the image of the graph also contains improvement ratios for other pairs of objectives which follow from the entered information. The file "direction setting interface.cdf" can be opened in any web browser and needs a free plug-in (if the plug-in is not installed, a link to it appears in the browser).

Let us illustrate the described procedure with a real-life problem presented in Cabello et al. (2014). The problem is to construct a portfolio of electrical power sources (electricity mix) for Andalucía region in Spain. Different ways of producing electricity have different impacts on economic and environmental interests of the region. We consider the following subset of seven objective functions (to be minimized) from the set of fourteen objective functions presented in the paper: Cost, annual cost of purchasing electricity $\left(f_{1}, \mathrm{M} €\right)$, Vulner, vulnerability of the production system measured as the share of imported fuel in the fuel consumption $\left(f_{2}, \%\right)$ and five environmental objectives estimating negative

\footnotetext{
${ }^{1}$ Note that any tree search algorithm can be used for discovering all vertices of this tree starting from $i$ or $j$. If the edge $\{i, j\}$ is not connected to any other existing edge, remove only pairs $(i, j)$ and $(j, i)$ from $P$.
} 


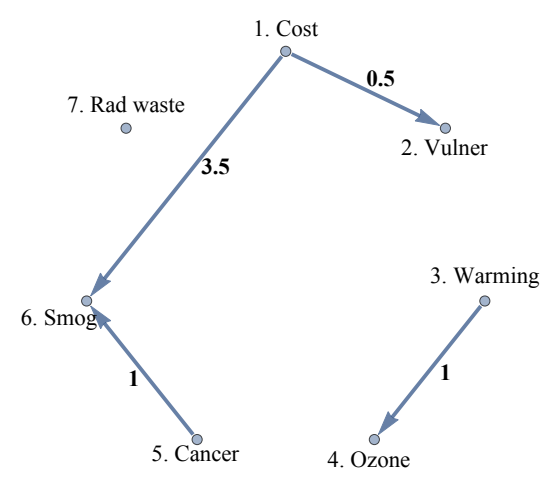

a

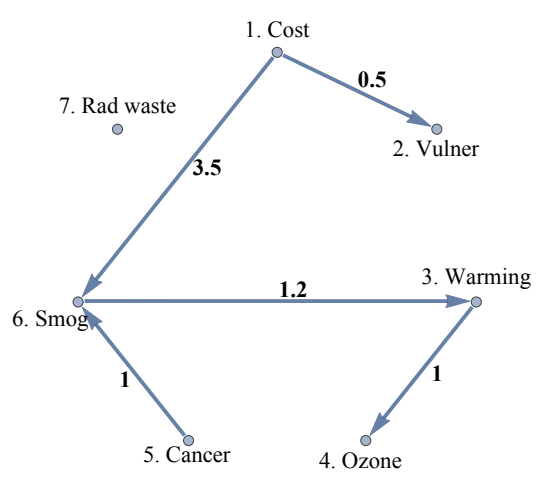

$\mathrm{b}$

Figure 1: Graph $G$ after the 4th and 5th iterations

effects of electricity production on the following aspects (each estimation is represented as a number of so-called Eco-points from 0 to 100): Warming, global warming $\left(f_{3}\right)$, Ozone, hole in the ozone layer $\left(f_{4}\right)$, Cancer, carcinogens $\left(f_{5}\right)$, Smog, winter smog $\left(f_{6}\right)$, Rad. waste, radioactive waste $\left(f_{7}\right)$.

We assume that the DM considers the objective vector $(3550,48 \%, 49,10,11.5,10$, 1.3) describing an unsatisfactory solution, and the direction of simultaneous improvement expresses the desire to improve the objective values. At the beginning of the procedure, we have $G=(V, \emptyset)$ where $V:=\{1, \ldots, 7\}$ corresponds to \{Cost, Vulner, Warming, Ozone, Cancer, Smog, Rad. waste $\}$, and $P=V \times V \backslash\{(1,1),(2,2), \ldots,(7,7)\}$.

1st iteration. The DM decided that saving each $\mathrm{M} €$ should be accompanied with a decrease of the share of imported fuel by $0.5 \%$. Then the improvement ratio between Cost and Vulner $\theta_{1,2}$ is set as $0.5, \theta_{2,1}$ is set as 2 and the edge $\{1,2\}$ is added to $G$. The pairs $(1,2)$ and $(2,1)$ are excluded from $\mathrm{P}$.

2nd and 3rd iterations. We assume that from the DM's point of view, in each of the following two pairs of objectives, both objectives should be improved in the same ratio: Warming and Ozone, and Cancer and Smog. This means setting $\theta_{3,4}=\theta_{5,6}=\theta_{4,3}=$ $\theta_{6,5}=1$ and adding edges $\{3,4\}$ and $\{5,6\}$ to $G$. Taking into account that these edges are not connected with any other edges of $G$, only pairs $(3,4),(4,3),(5,6)$ and $(6,5)$ are removed from $P$. Observe that after the 3rd iteration, $G$ consists of three disconnected trees of size 2 (disconnected edges), and an isolated vertex 7 .

4th iteration. We assume that the DM associates decreasing Cost by each $\mathrm{M} €$ with decreasing Smog by 3.5 Eco-points. Then $\theta_{1,6}$ is set to $3.5, \theta_{6,1}$ is set to $2 / 7$ and the edge $\{1,6\}$ is added to $G$ making its directed version look like in Figure 1 a. The pairs $(1,6)$, $(6,1),(1,5),(5,1),(2,6)$ and $(6,2)$ are removed from $P$.

5th iteration. The DM decides that decreasing Smog by each Eco-point should be accompanied by decreasing Warming by 1.2 Eco-points. Then $\theta_{6,3}$ is set to $1.2, \theta_{3,6}$ is set to $5 / 6$ and the edge $\{3,6\}$ is added to $G$ making its directed version look like in Figure 1 b. Now $P$ contains only the pairs $(1,7),(2,7), \ldots,(6,7)$ and $(7,1),(7,2), \ldots,(7,6)$.

6th iteration. The DM provides the improvement ratio between Rad. waste and Cancer as 2. Then $\theta_{7,5}$ is set to $2, \theta_{5,7}$ is set to 0.5 and the edge $\{5,7\}$ is added to $G$. After this, $G$ becomes a spanning tree of $K_{7}$ as the number of edges equals to 6 (see Figure 2). This means that the procedure of setting improvement ratios is completed.

Finally, we use Procedure 1 to obtain the direction of improvement, that is, we consider the tree shown in Figure 2 to conduct breadth-first search of all vertices starting from 1: 


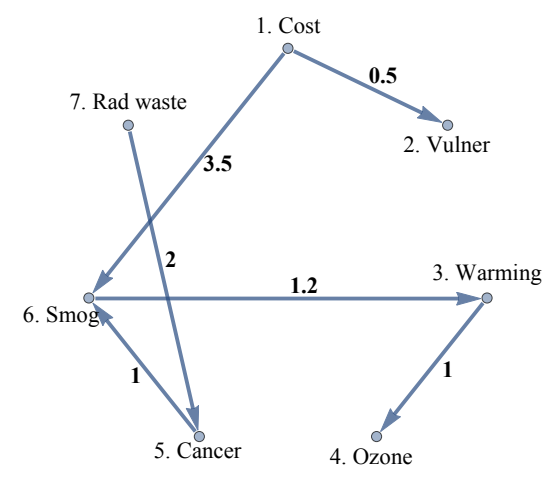

Figure 2: Graph $G$ built as a result of the procedure of setting improvement ratios

$\delta_{1}=1$.

1. From vertex 1 , visit $2\left(\delta_{2}=\delta_{1} * 0.5=0.5\right)$ and $6\left(\delta_{6}=\delta_{1} * 3.5=3.5\right)$.

2. From vertex 6 , visit $3\left(\delta_{3}=\delta_{6} * 1.2=4.2\right)$ and $5\left(\delta_{5}=\delta_{6} * 1=3.5\right)$.

3. From vertex 5 , visit $7\left(\delta_{7}=\delta_{5} / 2=1.75\right)$.

4. From vertex 3 , visit $4\left(\delta_{4}=\delta_{3} * 1=4.2\right)$.

As a result, we obtain the direction: $\delta=(1,0.5,4.2,4.2,3.5,3.5,1.75)$.

It is important to note that the final direction of improvement obtained using our new preference handling technique makes use of information that is not hard for the DM to provide. On the contrary, when using the percentage importance information used in Miettinen et al. (2010), in order to obtain weights following expression (3), which are equivalent to the direction $(1,0.5,4.2,4.2,3.5,3.5,1.75)$, the DM would have to be able to give directly the following percentages

$$
(2.21 \%, 22.08 \%, 18.54 \%, 18.54 \%, 15.45 \%, 15.45 \%, 7.73 \%)
$$

which cannot be regarded as an easy task. On the other hand, it is not at all possible to obtain this particular direction using integer ranks in (2). To get an equivalent rank the DM would have to use decimal ranks as follows

$$
J_{1}=\{1\}, J_{3.5}=\{7\}, J_{7}=\{5,6\}, J_{8.4}=\{3,4\}, J_{10}=\{2\} .
$$

Using decimal ranks is not allowed in Miettinen et al. (2010), as it is not realistic to assume that the DM could provide such information.

The percentages and ranks have been obtained by taking into account the ranges of the objective functions in the Pareto optimal set. As can be seen, a DM would hardly be able to provide such accurate information using the importance information required in Miettinen et al. (2010), and the results obtained using other importance information would most probably be misleading. An integer ranking close to the previous one, like for example,

$$
J_{1}=\{1\}, J_{4}=\{7\}, J_{7}=\{5,6\}, J_{8}=\{3,4\}, J_{10}=\{2\},
$$




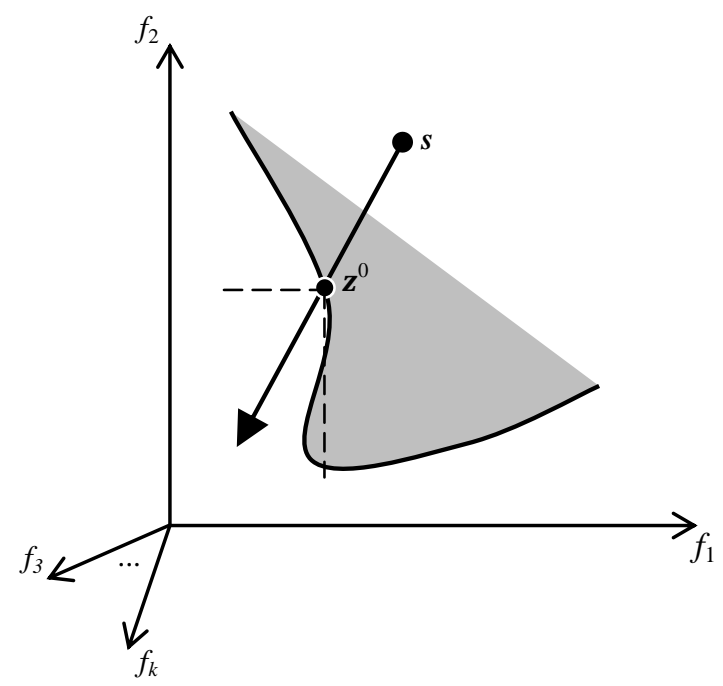

Figure 3: Objective vector $\mathbf{z}^{0}$ satisfying DM's preferences is not Pareto optimal, because it is dominated by other objective vectors (in the cone outlined by dashed lines).

can (depending on the structure of the Pareto optimal set) lead to a different solution. Besides, if the DM is thinking in terms of relative importance of the objectives, for the previous case (s)he would most probably give the following classes:

$$
J_{1}=\{1\}, J_{2}=\{7\}, J_{3}=\{5,6\}, J_{4}=\{3,4\}, J_{5}=\{2\},
$$

which is equivalent (taking $f_{1}$ as the reference function) to the direction of improvement

$$
\delta=(1,0.25,2,2,1.5,1.5,1) .
$$

As can be observed, the values assigned to functions $f_{2}-f_{6}$ are significantly smaller than those of the original direction. Therefore, this direction of improvement will probably produce worse results for these functions.

With the examples above we have demonstrated severe shortcomings of the ways of expressing preference information given in Miettinen et al. (2010). They imply only rough means of representing preference information. The new preference handling technique proposed enables a DM to conveniently and directly control how objective function values should be improved. It involves understandable questions and the preference information is directly applicable without any mappings to weights.

\subsection{Deriving solutions}

Next we address the issue of determining a solution which satisfies the DM's preferences expressed as a direction of simultaneous improvement of objectives. Improving objectives as much as possible starting from $\mathbf{s}$ in the direction $\delta$ literally means finding a feasible solution for which the corresponding objective vector is located farthest from $\mathbf{s}$ along the half line $\{\mathbf{s}-h \delta, h \geq 0\} \subset \mathbb{R}^{k}$ or, in other words, an objective vector which solves the following single objective optimization problem

$$
\max \{h: h \in \mathbb{R}, h>0, s-h \delta \in \mathbf{f}(S)\} .
$$

However, problem (4) is inappropriate as it does not necessarily have solutions (e.g., the half line does not necessarily intersect with the feasible objective set). Even if a 


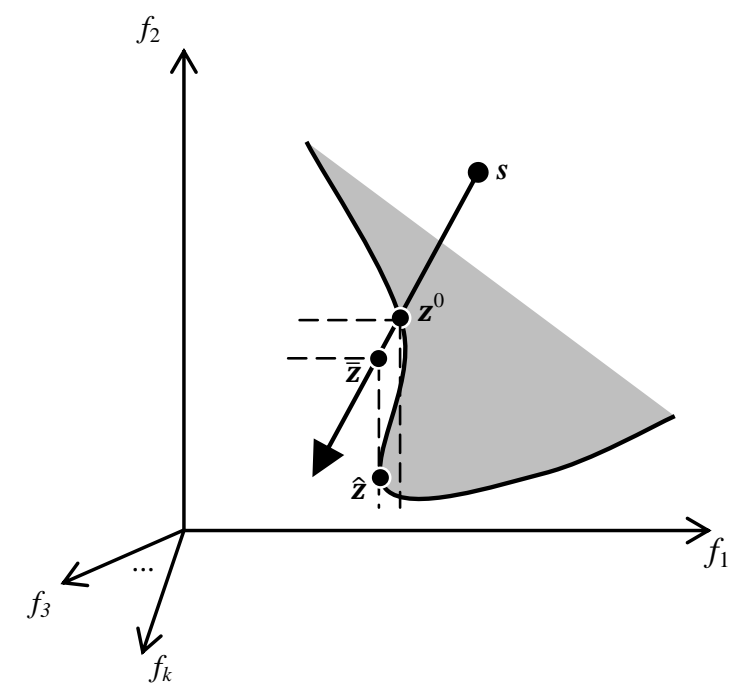

Figure 4: Selecting the solution $\hat{\mathbf{z}}$ satisfying the DM's preferences.

solution exists, it is not guaranteed to be Pareto optimal as, for example, in Figure 3. Note that even though we question the meaningfulness of employing only Pareto optimal solutions during the interactive solution process, we still expect the final solution to be Pareto optimal. Thus, we need a method that guarantees generating a Pareto optimal solution satisfying the DM's preferences. Such a method is described below.

If a solution to (4) exists, let us denote the corresponding objective vector by $\mathbf{z}^{0}$. Otherwise, we set $\mathbf{z}^{0}=\mathbf{s}$. If $\mathbf{z}^{0}$ is a Pareto optimal objective vector, we have found a feasible solution in the direction defined by $\delta$, which cannot be further improved. Therefore, we choose a feasible decision vector corresponding to $\mathbf{z}^{0}$ as the solution satisfying the DM's preferences. In the case $\mathbf{z}^{0}$ is not feasible or Pareto optimal, we proceed as follows. Given a point $\mathbf{z}$ on the line defined by the direction of improvement, let us call any Pareto optimal objective vector dominating $\mathbf{z}$ as its superior. Let us move from $\mathbf{z}^{0}$ along the direction of improvement until we find the farthest point in this direction having a superior. We denote this farthest point by $\overline{\mathbf{z}}$. We select any objective vector $\hat{\mathbf{z}}$ among superiors of $\overline{\mathbf{z}}$ as the solution satisfying the DM's preferences.

Figure 4 illustrates this solution selection reasoning in the case where $\mathbf{z}^{0}$ is not Pareto optimal. Observe that $\hat{\mathbf{z}}$ does not lie on the half line defined by the desired direction of improvement. However, the correspondence of the derived solution to the DM's preferences can be justified: $\overline{\mathbf{z}}$ is preferred to $\mathbf{z}^{0}$ because it lies farther along the direction of improvement, and $\hat{\mathbf{z}}$ is preferred to $\overline{\mathbf{z}}$ because of domination. No further improvement of the objective vector based on the available preference information can be obtained.

To derive a solution which satisfies the DM's preferences according to the above reasoning, we solve the following problem involving an augmented achievement scalarizing function (from Wierzbicki (1986))

$$
\min _{\mathbf{x} \in S} \max _{i=1, \ldots, k} \frac{1}{\delta_{i}}\left(f_{i}(\mathbf{x})-s_{i}\right)+\rho \sum_{j=1, \ldots, k}\left(f_{j}(\mathbf{x})-s_{j}\right),
$$

where $\rho>0$ is a small augmentation coefficient. It can be proven that by solving this problem one obtains a Pareto optimal solution (see, e.g., Miettinen (1999); Wierzbicki (1986)). In our case, it corresponds to the preference information expressed as the direc- 
tion of simultaneous improvement of objectives, where the correspondence is understood through the reasoning above.

It is important to note that as has been proved in Wierzbicki (1986), all properly Pareto optimal solutions and only them can be obtained by solving (5) with different parameters. The set of properly Pareto optimality is a subset of Pareto optimal solutions (see e.g. Miettinen (1999)) where $\rho$ sets bounds on trade-offs (for more details, see e.g. Wierzbicki (1986); Podkopaev (2007)). Because we do not consider bounding trade-offs, we set $\rho$ as a small positive value which ensures proper Pareto optimality, but does not significantly influence the properties of solutions obtained.

Originally, problem (5) was formulated for deriving solutions based on preference information expressed as aspiration levels of objectives in the reference point method, see Wierzbicki (1980, 1986) and Miettinen (1999). The main differences in our approach are the way how DM's preferences are elicited and how the solution selection process is interpreted. In reference point based methods, a solution closest (in some sense) to the reference point is searched for. In our approach, based on a direction of improvement, the reference point is replaced by the starting point which has a different meaning. (Note that achievement scalarizing functions have other interpretations related to a DM's preference models see, e.g., Kaliszewski (2004); Luque et al. (2009).)

\section{Examples of Applying the New Preference Handling Tech- nique}

In this section, we give two examples of how the new preference handling technique proposed in the previous section can be applied. In the first example, we describe how the technique can be incorporated into an interactive method without trading-off. In the second subsection, we demonstrate how this method can be applied, that is, we describe the solution process of solving a numerical example.

\subsection{Algorithm Example: NAUTILUS 2}

In this section, we integrate the new preference handling technique into an interactive method which does not require the DM to consider Pareto optimal solutions until the solution process is at the very end. The method we present is called NAUTILUS 2, and it is a modification of the NAUTILUS method proposed in Miettinen et al. (2010). In the following paragraphs, for completeness, we briefly describe the main features of the method.

NAUTILUS 2 adopts the core idea of NAUTILUS which is to iteratively direct the search starting from an inferior point towards the most preferred solution by simultaneously improving all objective function values. The solution derived and shown to the DM at each iteration dominates that of the previous iteration. Thus, by starting from an inferior point the DM can reach any Pareto optimal solution dominating this point and, in particular, by starting from the nadir objective vector the DM can reach any Pareto optimal solution. Note that after each iteration, the set of reachable Pareto optimal solution narrows. However, this narrowing is reversible as the DM is allowed to make steps backwards by returning to solutions from previous iterations. At each iteration, the DM can get information about the reachable part of the Pareto optimal set and the proximity to it and, thus, gain understanding of which parts of the Pareto optimal set can no longer be obtained without going backwards and what can be achieved when approaching the Pareto optimal set in a certain direction. 
It must be pointed out that the idea of finding a Pareto optimal solution as the final one is not questioned by NAUTILUS 2. Moreover, the DM does not lose sight of the Pareto optimal set at any moment during the solution process. In this way, NAUTILUS 2 supports the DM in a free search for the most preferred solution. Next we describe the NAUTILUS 2 method.

In the initialization step, the DM is asked to determine the starting objective vector $\mathbf{z}^{0}$. It has the same meaning as the starting point $\mathbf{s}$ described in Section 3, i.e., it is an inferior objective vector which the DM wishes to improve during the solution process. For example, one can set $\mathbf{z}^{0}=\mathbf{z}^{\text {nad }}$. The DM can also optionally give the overall number of iterations (s)he wishes to carry out, denoted by itn. This initial estimate is not a critical parameter, because it can be modified at any time during the solution process if the DM wishes so.

We denote by $h$ the current iteration (ordinary) number, and by $\mathbf{z}^{h}$ the vector in the objective space corresponding to the current iteration to be called the current iteration point. If the desirable number of iterations was provided by the DM, we denote by $i t^{h}$ the number of iterations left (including iteration $h$ ). Therefore, $i t^{1}=i t n$.

To proceed to the next iteration, a Pareto optimal solution $\mathbf{x}^{h}$ is generated by solving (5) based on the preference information obtained from the DM using the preference handling technique described in Section 3. Because the previous iteration point $\mathbf{z}^{h-1}$ is used as the starting point $\mathbf{s}$, the DM provides only the information about the direction of improvement $\delta$. We denote $\mathbf{f}^{h}=\mathbf{f}\left(\mathbf{x}^{h}\right)$. The current iteration point $\mathbf{z}^{h}$ will lie on the segment between the previous iteration point $\mathbf{z}^{h-1}$ and $\mathbf{f}^{h}$. Its exact location is determined via a parameter $v, 0<v \leq 1$, representing the ratio between the distance from $\mathbf{z}^{h-1}$ to $\mathbf{z}^{h}$ and from $\mathbf{z}^{h-1}$ to $\mathbf{f}^{h}$ :

$$
\mathbf{z}^{h}=(1-v) \mathbf{z}^{h-1}+v \mathbf{f}^{h} .
$$

One should observe that $\mathbf{z}^{h}$ dominates $\mathbf{z}^{h-1}$. In this way, the DM gets closer to the Pareto optimal set. The larger the value of $v$, the faster the DM approaches the Pareto optimal set. Therefore, it is reasonable to adjust the rule of setting the value of $v$ so that the pace of movement towards the Pareto optimal set is suitable for the DM. For example, some DMs may wish to take relatively big steps at the beginning of the solution process and move with more care closer to the end. Then, a "rule of thumb" can be to set $v=1 / 2$. If the desirable number of iterations is given by the DM, the parameter $v$ can be defined as proposed in Miettinen et al. (2010):

$$
v=\frac{1}{i t^{h}}
$$

which means that at each iteration, the relative amount of movement towards the Pareto optimal set increases. It must be noted that if $h$ is the last iteration, then $i t^{h}=1$, and thus, $\mathbf{z}^{h}=\mathbf{f}^{h}$ is Pareto optimal and the final objective vector, while $\mathbf{x}^{h}$ is the corresponding final solution in the decision space.

A graphical idea of the solution process is shown in Figure 5, where the desirable number of iterations is four, and two iterations have been taken. At each iteration, the current iteration point $\mathbf{z}^{h}$ is a point lying in the segment that joins $\mathbf{z}^{h-1}$ and $\mathbf{f}^{h}$. It gets closer to the Pareto optimal set as the iterations proceed.

The information about Pareto optimal objective vectors that are reachable starting from the current iteration point is provided to the DM in terms of lower and upper bounds on vectors' components. These bounds are denoted by $\mathbf{z}^{h, l o}$ and $\mathbf{z}^{h, u p}$, respectively. It is obviously that $\mathbf{z}^{h, u p}=\mathbf{z}^{h-1}$. The $r$-th component of $\mathbf{z}^{h, l o}, r=1, \ldots, k$, is obtained as the optimal objective function value of a corresponding $\varepsilon$-constraint problem $\left(P_{r}^{h}\right)$ (see Miettinen et al., 2010). 


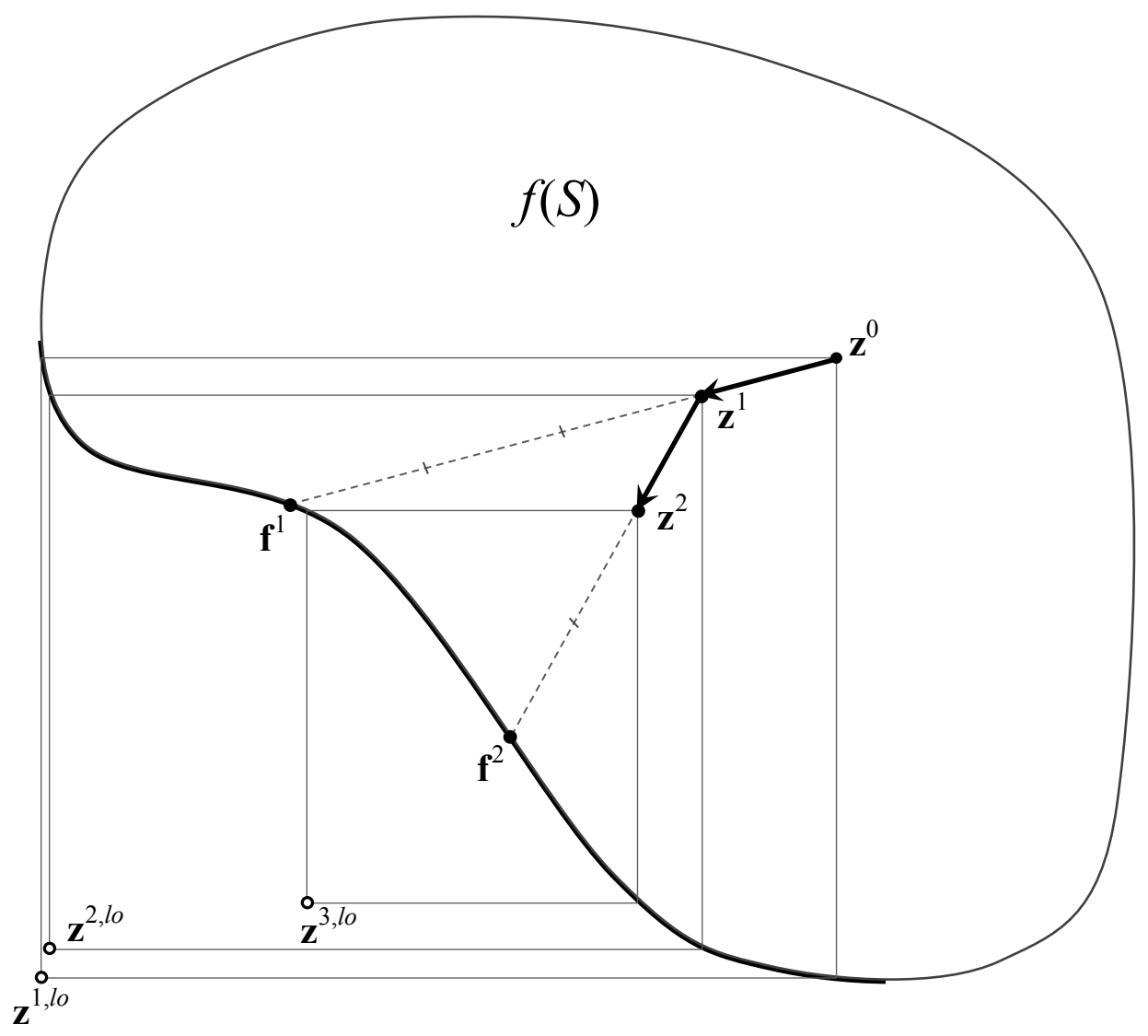

Figure 5: Graphical idea of the NAUTILUS 2 method.

It is verified that for each iteration $h,\left[z_{i}^{h, l o}, z_{i}^{h, u p}\right] \subseteq\left[z_{i}^{h-1, l o}, z_{i}^{h-1, u p}\right]$. This means that once a Pareto optimal solution is left out of the box defined by $\mathbf{z}^{h, l o}$ and $\mathbf{z}^{h, u p}$, it will not be reachable at any following iteration, unless a step backwards is taken. For this reason, the attainable ranges of objective function values provide very useful information to the DM, because they allow to evaluate the effect of the current iteration, and to decide whether to carry on or to go backwards.

Note that the computational cost of solving the $k$ problems $\left(P_{r}^{h}\right)$ at each iteration may be high for some problems like simulation-based optimization problems. Therefore, depending on the special features of each particular problem, one must decide whether these bounds are worth being calculated, or whether it is too time-consuming.

Another piece of information to be shown to the DM is the relative closeness of the current iteration point to the Pareto optimal set. We propose to use the same approach to calculating the measure of the closeness as in Miettinen et al. (2010):

$$
d^{h}=\frac{\left\|\mathbf{z}^{h}-\mathbf{z}^{0}\right\|_{2}}{\left\|\mathbf{f}^{h}-\mathbf{z}^{0}\right\|_{2}} \times 100,
$$

where $\|\cdot\|_{2}$ is the $L_{2}$-norm, that is, for $\mathbf{z}=\left(z_{1}, \ldots, z_{k}\right)^{T} \in \mathbb{R}^{k},\|\mathbf{z}\|_{2}=\sqrt{z_{1}^{2}+\cdots+z_{k}^{2}}$. Note that if $\mathbf{z}^{h}=\mathbf{z}^{0}$, then $d^{h}=0$ and, on the other hand, if $\mathbf{z}^{h}=\mathbf{f}^{h}$, then $d^{h}=100$.

Summing up, after having specified preference information with the new preference handling technique, at each iteration of the NAUTILUS 2 method, the DM directs the solution process by choosing between the following options:

- take a step forward without changing the preference information; 
- take a step forward providing new preference information to change the direction;

- take a step backwards;

- stop the solution process.

Besides this, at each iteration, the DM may ask to increase or decrease the pace of movement towards the Pareto optimal set by changing $v$, or redefine the number of remaining iterations, if it was given. The solution process continues until the most preferred Pareto optimal solution is found. The NAUTILUS 2 method is described in detail in Appendix 1.

Unlike the original NAUTILUS method (as criticized in Section 3), NAUTILUS 2 provides a trade-off-free decision making approach which is transparent to the DM. To be more specific, the DM can directly control the solution process by giving directions of simultaneous improvement of objective functions rather than considering the importance of improving different objectives, which requires mappings and enables controlling the solution process only roughly.

Apart from solving multiobjective optimization problems, a natural field of application of NAUTILUS 2 is negotiation support. As it was mentioned before, starting from a Pareto optimal solution in a group decision making problem is not always a good policy. When several DMs with conflictive objectives negotiate, this can result in anchoring for those negotiators who regard the current Pareto optimal solution as advantageous for their interests. Therefore, starting from a "bad" point and moving towards a solution that dominates it (so-called win-win situation) enables the DMs to continue negotiating, because they all know that they can improve their payoffs.

In group decision making problems, negotiations can consist of determining the starting point $\mathbf{s}$ and the directions of improvement $\delta$. Such negotiations may exhibit much smaller degrees of conflict and anchoring effects than negotiations over Pareto optimal objective function values, as the notions of a starting point and a direction of improvement can be regarded by the DMs as less straightforward payoffs than objective vectors themselves. Note that introducing negotiations into the process of determining the direction of improvement may require further developing the preference elicitation techniques described in Section 3.

\subsection{Numerical Example}

In this section, we demonstrate how NAUTILUS 2 involving the new preference handling technique can be applied to solve a multiobjective optimization problem. We consider a situation where a group of DMs solves a problem, where no a priori information is available about the structure of the Pareto optimal set or trade-offs between objective functions. We consider as a group decision making problem the problem of designing water treatment facilities presented in Narula and Weistroffer (1989) (originally for a single DM).

There are three objects located in a valley along a river: Fresh Fishery Company near the head of the valley, the city of Fortuna downstream from the fishery, and the municipality border downstream from the city. The company and the city are sources of river pollution resulting in a low dissolved oxygen level (DOL) in the water measured in $\mathrm{mg} / \mathrm{l}$. The company and the city are planning a joint investment to build water treatment facilities along the river. They need to decide on treatment facilities which influence the improvement of water quality (in terms of increasing DOL) and costs. The latter in turn affects the financial performance of the company and the city.

We assume that there are three parties (DMs) participating in the solution process: 
(1) association "Citizens for clear water",

(2) the Fresh Fishery Company and

(3) the city council.

The following four objectives are considered:

$f_{1}$ maximize DOL in the city,

$f_{2}$ maximize DOL at the municipality border,

$f_{3}$ maximize the percent return on equity at Fresh Fishery and

$f_{4}$ minimize addition to the tax rate at Fortuna.

It is natural to assume that not all the DMs are equally interested in each objective: for example, (1) is interested in $f_{1}$ and $f_{4},(2)$ is mostly interested in $f_{3}$ and slightly in $f_{4}$, and $(3)$ is interested in $f_{1}, f_{2}$ and $f_{4}$.

The following problem was presented in Narula and Weistroffer (1989) (converting all the objectives to be minimized)

$$
\begin{array}{ll}
\operatorname{minimize} & f_{1}(\mathbf{x})=-4.07-2.27 x_{1} \\
& f_{2}(\mathbf{x})=-2.60-0.03 x_{1}-0.02 x_{2}-\frac{0.01}{1.39-x_{1}^{2}}-\frac{0.30}{1.39-x_{2}^{2}} \\
& f_{3}(\mathbf{x})=-8.21+\frac{0.71}{1.09-x_{1}^{2}} \\
& f_{4}(\mathbf{x})=-0.96+\frac{0.96}{1.09-x_{2}^{2}} \\
\text { subject to } & 0.3 \leq x_{i} \leq 1.0, \text { for } i=1,2,
\end{array}
$$

where the decision variables $x_{1}$ and $x_{2}$ determine proportional amounts of two types of contaminants removed from the water. An example of a solution process utilizing the NAUTILUS 2 method and the directions of simultaneous improvement of objectives is described below.

Initialization

The starting point $\mathbf{z}^{0}$ is chosen as follows. Its first and second components are set to the current DOLs at the city and the municipality border, respectively. These levels can be determined as DOLs in the case were no contaminants are removed, i.e., $z_{1}^{0}=$ $f_{1}(0,0)=-4.07$ and $z_{2}^{0}=f_{2}(0,0)=-2.82$. The fishery agrees to take part in the project under the condition that the return on equity will not fall below $3 \%$, therefore $z_{3}^{0}=-3$. The city determines the maximum allowable tax increase as $4 \%$, therefore $z_{4}^{0}=4$. Thus, $\mathbf{z}^{0}=(-4.07,-2.82,-3,4)^{T}$. The value of $\rho$ is set to $10^{-5}$. (The starting point is such that all objective function values can be improved.)

By solving four $\varepsilon$-constraint problems $\left(P_{r}^{1}\right)$ we obtain $\mathbf{z}^{1, l o}=(-6.29,-3.28,-7.5,0)^{T}$. This information is presented to the DMs. They agree to set the maximum number of iterations to three (and thereby the velocity $v$ is set by (7)).

1st iteration

The DMs start negotiating on the direction of improvement.

- The city council proposes to start from the following direction of improvement: $\delta_{1}=$ $1.5 \mathrm{mg} / 1, \delta_{2}=2 \mathrm{mg} / \mathrm{l}, \delta_{3}=0.5 \mathrm{pp}$ (percentage points) and $\delta_{4}=1 \mathrm{pp}$.

- The association "Citizens for clear water" insists that clear water at the city level is more important than at the municipality border. Thus, it proposes to increase $\delta_{1}$ to $3: \delta=(3,2,0.5,1)^{T}$. 
- The Fresh Fishery representative indicates that when compared to $\delta_{1}$ and $\delta_{2}$, the value of $\delta_{3}$ is too small, while the fishery provides half of the invested money. Thus, the following requirements should be met: $\frac{\delta_{3}}{\delta_{1}} \geq 0.5, \frac{\delta_{3}}{\delta_{2}} \geq 0.5$ and $\frac{\delta_{3}}{\delta_{4}} \geq 0.75$. The representative proposes to increase $\delta_{3}$ to 1.5 and we get $\delta=(3,2,1.5,1)^{T}$.

- The City Council, emphasizing financial aspects, proposes to decrease $\delta_{1}$ and $\delta_{2}$ by one, which does not violate conditions imposed by two other DMs: $\delta=(2,1,1.5,1)^{T}$. Thus, the increase of DOL at the municipality border by each unit (mg/l) should be accompanied with the increase of DOL in the city by $2 \mathrm{mg} / \mathrm{l}$, increase of the percent return at Fresh Fishery by $1.5 \%$ and decrease of city taxes by $1 \%$.

With $\delta=(2,1,1.5,1)^{T}$ as the final result of negotiations at the 1 st iteration and solving (5) with $\mathbf{s}=\mathbf{z}^{0}=(-4.07,-2.82,-3,4)^{T}$ and $\delta=(2,1,1.5,1)^{T}$ gives a Pareto optimal objective vector $\mathbf{f}^{1}=(-6.26,-3.26,-3.65,3.57)^{T}$. The iteration point $\mathbf{z}^{1}=$ $(-4.8,-2.97,-3.22,3.86)^{T}$ is computed by $(6)$. The lower bound vector for the next iteration is found to be $\mathbf{z}^{2, l o}=(-6.28,-3.27,-7.5,0.43)^{T}$. Vectors $\mathbf{z}^{1}$ and $\mathbf{z}^{2, l o}$ are presented to the DMs. They are also informed that the closeness of the current iteration point to the Pareto optimal set calculated by (8) is equal to $33.3 \%$.

\section{2nd iteration}

When analyzing $\mathbf{z}^{1}$ and $\mathbf{z}^{2, l o}$, the DMs notice that there is not much space for further improvement of water quality, while financial indicators have been noticeably improved and there is some potential for further improvement. Therefore, it is decided to give more emphasis to improving the water quality by increasing $\delta_{1}$ and $\delta_{2}$ by 1 leaving $\delta_{3}$ and $\delta_{4}$ on the same levels.

Solving (5) with $\mathbf{s}=\mathbf{z}^{1}=(-4.8,-2.97,-3.22,3.86)^{T}$ and $\delta=(3,2,1.5,1)^{T}$ gives $\mathbf{f}^{2}=$ $(-6.27,-3.26,-3.44,3.71)^{T}$. The next iteration point $\mathbf{z}^{2}=(-5.54,-3.12,-3.33,3.78)^{T}$ and the lower bound for the next iteration $\mathbf{z}^{3, l o}=(-6.28,-3.27,-7.15,1.62)^{T}$ are calculated and presented to the DMs. They are also informed that the closeness of the current iteration point to the Pareto optimal set now equals $56.1 \%$.

\section{3rd (final) iteration}

The DMs are quite satisfied with the improvement of DOL levels and want to set more emphasis on financial performance. The Fresh Fishery representative has been convinced that decreasing taxes is more important, as it will also influence the Fresh Fishery's financial situation. Therefore, the DMs agree that further increase of the percent return on equity at Fresh Fishery has to be accompanied with twice the decrease of additional tax rate at Fortuna. As a result, the following direction of improvement is accepted: $\delta=(2,1,5,10)^{T}$.

Solving (5) with $\mathbf{s}=\mathbf{z}^{2}=(-5.54,-3.12,-3.33,3.78)^{T}$ and $\delta=(2,1,5,10)^{T}$ gives the final Pareto optimal solution $\mathbf{x}^{*}=(0.964,0.9146)^{T}$ corresponding to the objective vector $\mathbf{f}^{3}=(-6.26,-3.21,-3.81,2.83)^{T}$.

For simplicity, we carried out only three iterations and did not take steps backwards. Our aim was to demonstrate that guiding the solution process based on the direction of improvement concept can be useful even in the case of group decision making. Each DM could attain improvement in one's objectives and direct the search for the final solution.

\section{Conclusions}

In this paper we have proposed a new preference handling technique for multiobjective optimization. Our research is intended to overcome cognitive difficulties a DM may face when solving multiobjective optimization problems by traditional methods, i.e., methods 
based on evaluating and comparing different Pareto optimal solutions during the solution process. Our idea is to protect the DM from the necessity of trading-off, always placing the DM in a situation where all the objectives can be improved simultaneously. In group decision making, this is referred to as a win-win situation. From ratios of improvement we get a direction of simultaneous improvement in the new preference handling technique which allows the DM to conveniently control how different objective functions should be improved.

In order to demonstrate the validity of the new preference handling technique, we have incorporated it in the earlier NAUTILUS method resulting with an interactive multiobjective optimization method NAUTILUS 2 which is much more convenient for the DM to provide preference information and directly control the solution process. The preference handling technique and the progress of the solution process do not force the DM into considering trade-offs.

The information about the DM's preferences in our method is presented in such a transparent form that it can be easily explained and discussed with others. Therefore, besides supporting a single DM in finding the most preferred solution without trading-off and anchoring, the proposed method can successfully be used in group decision making, where at each iteration several DMs negotiate on the direction of simultaneous improvement of objectives.

We have demonstrated the course of such a group decision making process with a numerical example. We believe that the new way of specifying preferences provides for DMs better control of directing solution processes as well and hinders premature convergence.

\section{Acknowledgements}

This research has been partially supported by the Regional Government of Andaluca (research groups SEJ-445 and SEJ-532), and by the Spanish Ministry of Education and Science (projects ECO2013-47129-C4-2 and MTM2010-14992).

\section{References}

Aloysius, J. A., Davis, F. D., Wilson, D. D., Taylor, A. R., and Kottemann, J. E. (2006). User acceptance of multi-criteria decision support systems: the impact of preference elicitation techniques. European Journal of Operational Research, 169, 273-285.

Arbel, A. and Korhonen, P. (1996). Using aspiration levels in an interior primal-dual multiobjective linear programming algorithm. Journal of Multi-Criteria Decision Analysis, $\mathbf{5}, 61-71$.

Branke, J., Deb, K., Miettinen, K., and Słowiński, R., editors (2008). Multiobjective Optimization: Interactive and Evolutionary Approaches, Berlin, Heidelberg. Springer.

Buchanan, J. T. and Corner, J. (1997). The effects of anchoring in interactive MCDM solution methods. Computers \& Operations Research, 24(10), 907-918.

Cabello, J., Luque, M., Miguel, F., Ruiz, A., and Ruiz, F. (2014). A multiobjective interactive approach to determine the optimal electricity mix in Andalucía (Spain). TOP, 22(1), 109-127.

Cormen, T. (2009). Introduction to Algorithms. Mit Press. 
Deb, K., Miettinen, K., and Chaudhuri, S. (2010). Towards an estimation of nadir objective vector using a hybrid of evolutionary and local search approaches. IEEE Transactions on Evolutionary Computation, 14(6), 821-841.

Ehtamo, H., Kettunen, E., and Hamalainen, R. P. (2001). Searching for joint gains in multi-party negotiations. European Journal of Operational Research, 130(1), 54-69.

Gardiner, L. and Vanderpooten, D. (1997). Interactive multiple criteria procedures: some reflections. In J. Climaco, editor, Multicriteria Analysis, pages 290-301, Berlin, Heidelberg. Springer.

Guerraggio, A. and Molho, E. (2004). The origins of quasi-concavity: a development between mathematics and economics. Historia Mathematica, 31(1), 62-75.

Janis, I. L. and Mann, L. (1977). Decision Making: a Psychological Analysis of Conflict, Choice and Commitment. The Free Press, New York.

Kahneman, D. and Tversky, A. (1979). Prospect theory: an analysis of decision under risk. Econometrica, 47(2), 263-291.

Kaliszewski, I. (2004). Out of the mist-towards decision-maker-friendly multiple criteria decision making support. European Journal of Operational Research, 158(2), 293-307.

Keeney, R. (1996). Value-Focused Thinking. A Path to Creative Decisionmaking. Harvard University Press, Cambridge, MA.

Korhonen, P. and Wallenius, J. (1996). Behavioural issues in MCDM: neglected research questions. Journal of Multi-Criteria Decision Analysis, 5, 178-182.

Luque, M., Miettinen, K., Eskelinen, P., and Ruiz, F. (2009). Incorporating preference information in interactive reference point methods for multiobjective optimization. Omega, 37(2), 450-462.

Luque, M., Ruiz, F., and Miettinen, K. (2011). Global formulation for interactive multiobjective optimization. OR Spectrum, 33, 27-48.

Miettinen, K. (1999). Nonlinear Multiobjective Optimization. Kluwer Academic Publishers, Boston.

Miettinen, K. (2002). Interactive nonlinear multiobjective procedures. In M. Ehrgott and X. Gandibleux, editors, Multiple Criteria Optimization: State of the Art Annotated Bibliographic Surveys, pages 227-276. Springer.

Miettinen, K. and Hakanen, J. (2009). Why use interactive multi-objective optimization in chemical process design? In G. P. Rangaiah, editor, Multi-Objective Optimization: Techniques and Applications in Chemical Engineering. World Scientific.

Miettinen, K., Ruiz, F., and Wierzbicki, A. (2008). Introduction to multiobjective optimization: interactive approaches. In J. Branke, K. Deb, K. Miettinen, and R. Słowiński, editors, Multiobjective Optimization: Interactive and Evolutionary Approaches, pages 27-57, Berlin, Heidelberg. Springer.

Miettinen, K., Eskelinen, P., Ruiz, F., and Luque, M. (2010). NAUTILUS method: an interactive technique in multiobjective optimization based on the nadir point. European Journal of Operational Research, 206(2), 426-434. 
Narula, S. C. and Weistroffer, H. R. (1989). A flexible method for nonlinear multicriteria decisionmaking problems. IEEE Transactions on Systems, Man, and Cybernetics, 19(4), 883-887.

Podkopaev, D. (2007). An approach to finding trade-off solutions by a linear transformation of objective functions. Control and Cybernetics, 36(2), 347-356.

Podkopaev, D. and Miettinen, K. (2011). Handling preferences in the "pre-conflicting" phase of decision making processes under multiple criteria. In R. Brafman, F. Roberts, and A.Tsoukias, editors, Algorithmic Decision Theory: Second International Conference, ADT 2011, Proceedings, pages 234-246, Berlin, Heidelberg. Springer.

Ruiz, F., Luque, M., and Miettinen, K. (2012). Improving the computational efficiency of a global formulation (GLIDE) for interactive multiobjective optimization. Annals of Operations Research, 197(1), 47-70.

Simon, H. A. (1956). Rational choice and the structure of the environment. Psychological Review, 63(2), 129-138.

Szczepanski, M. and Wierzbicki, A. P. (2003). Application of multiple criterion evolutionary algorithm to vector optimization, decision support and reference point approaches. Journal of Telecommunications and Information Technology, 3, 16-33.

Tsay, C.-J. and Bazerman, M. H. (2009). A decision-making perspective to negotiation: a review of the past and a look to the future. Negotiation Journal, 25(4), 467-480.

Wierzbicki, A. P. (1980). The use of reference objectives in multiobjective optimization. In G. Fandel and T. Gal, editors, Multiple Criteria Decision Making: Theory and Application, pages 468-486, Berlin, Heidelberg. Springer.

Wierzbicki, A. P. (1986). On the completeness and constructiveness of parametric characterizations to vector optimization problems. OR Spectrum, 8, 73-87.

\section{Appendix 1}

Algorithm of the NAUTILUS 2 method.

Step 0. Initialization. Ask the DM to determine the starting point $\mathbf{z}^{0}$. Alternatively, estimate the nadir objective vector and set it as $\mathbf{z}^{0}$. Set $h=1$ and $\rho$ to a small positive scalar. Calculate lower bounds $\mathbf{z}^{1, l o}$ for the first iteration by solving the $k$ problems $\left(P_{r}^{h+1}\right)$. If the DM wishes to give the number of iterations itn to be carried out, set $i t^{1}=i t n$ and set $v$ according to (7), otherwise, set $v=1 / 2$.

Step 1. Preference information I. Elicit from the DM preference information in terms of proportions of simultaneous improvement of objectives $\delta_{i}^{h}(i=1, \ldots, k)$ using one of three approaches (i)-(iii) described in Section 3.

Step 2. New solution. Set $\mathbf{s}=\mathbf{z}^{h-1}$ and $\delta_{i}=\delta_{i}^{h}(i=1, \ldots, k)$ and solve problem (5). Let $\mathbf{x}^{h}$ be the optimal solution and $\mathbf{f}^{h}$ the corresponding objective vector.

Step 3. New iteration point. Calculate a new iteration point $\mathbf{z}^{h}$ according to (6).

Step 4. Bounds for the next iterations. Given $\mathbf{z}^{h}$, find the vector $\mathbf{f}^{h+1, l o}$ by solving the $k$ problems $\left(P_{r}^{h+1}\right)$. Furthermore, set $\mathbf{f}^{h+1, u p}=\mathbf{z}^{h}$. Calculate the distance to the Pareto optimal set $d^{h}$ using formula (8). 
Step 5. Show current iteration point. Show the current values $z_{i}^{h}(i=1, \ldots, k)$, together with the additional information $\left[z_{i}^{h+1, l o}, z_{i}^{h+1, u p}\right](i=1, \ldots, k)$ and $d^{h}$ to the DM.

Step 6. Revise the approach rhythm. If the DM wishes to speed up or slow down the pace of moving toward the Pareto optimal set, adjust the parameter $v$ used in (6). Namely, if $v$ was originally set to $1 / 2$, multiply $v$ by 2 (speed up) or divide by 2 (slow down). If the desirable number of iterations was set by the DM, (s)he must set a new value for the number of remaining iterations and $i t^{h}$ is set accordingly.

Step 7. Preference information II. Ask the DM whether (s)he would like to take a step backwards. If so, go to step 9. Otherwise, continue.

Step 8. Next iteration. If $i t^{h}=1$ or the DM wishes to stop, stop the solution process with the last solution $\mathbf{x}^{h}$ and the corresponding objective vector $\mathbf{f}^{h}$ as the final Pareto optimal solution. Otherwise, set $h=h+1$ and $i t^{h+1}=i t^{h}-1$, if $i t^{h}$ is set. If the DM wants to give new preference information, go to step 1. Alternatively, the DM can take a new step in the same direction (using the preference information of the previous iteration). Then, set $\mathbf{f}^{h}=\mathbf{f}^{h-1}$, and go to step 3 .

Step 9. Preference information III. Ask the DM whether (s)he would like to provide new preference information starting from the iteration point $\mathbf{z}^{h-1}$. If so, go to step 1. Alternatively, the DM can take a shorter step from the previous iteration point with the same preference information given in step 1 . Then, set $\mathbf{z}^{h}=\frac{1}{2} \mathbf{z}^{h}+\frac{1}{2} \mathbf{z}^{h-1}$ and go to step 4 . 